\section{VACCINATING CHILDREN IN THE EMERGENCY DEPARTMENT, A NOVEL WAY TO INCREASE INFLUENZA VACCINATION RATES}

${ }^{1}$ Shannon Baumer-Mouradian, ${ }^{2}$ Abigail Kleinschmidt, ${ }^{2}$ Ashley Servi, ${ }^{2}$ Brian Jaworski, ${ }^{2}$ Kimberly Lazarevic, ${ }^{2}$ Matthew Kopetsky, ${ }^{1}$ Mark Nimmer, ${ }^{2}$ Thomas Hanson, ${ }^{1}$ Matthew Gray, ${ }^{1}$ Amy Drendel. 'Medical College of Wisconsin, United States; ${ }^{2}$ Children's Hospital of Wisconsin, United States

\subsection{6/bmjoq-2019-ihi.4}

Background Universal administration of the influenza vaccine is the best strategy for preventing morbidity and mortality. Vaccine administration in the emergency department (ED) is an innovative approach to improving vaccination rates. However, during the 2017-2018 influenza season only 75 influenza vaccines were administered, $0.2 \%$ of the total population in this tertiary care ED.

Objectives We aimed to increase the number of influenza vaccines administered from 75 to 1000 in patients discharged from the ED between September 2018 and March 2019.

Methods A multidisciplinary workgroup performed process mapping to identify potential barriers and solutions. The electronic health record was engineered to facilitate our key interventions: a required influenza vaccination screening question, scripted patient education, and an influenza vaccine nursing protocol with a clinical decision support tool. Interventions were tested using plan-do-study-act cycles. Process measures included percent screened and percent requested. The primary outcome was the number of influenza vaccines administered. The balancing measure was ED length of stay.

Results 33,311 ED patients met inclusion criteria. In this population, influenza vaccination screening rates improved from 0 to $90 \%$ (figure 1) and $41 \%$ of children were found to be unvaccinated. 1323 children (4\%) received the influenza vaccine (figure 2). Additionally, there was no change in ED LOS (139 min).

Conclusions An efficient, nurse-driven workflow that screened for vaccination status, educated parents, and then provided the influenza vaccination to children during the ED visit significantly increased the number of children receiving the influenza vaccine from 75 to 1323 without extending length of stay.

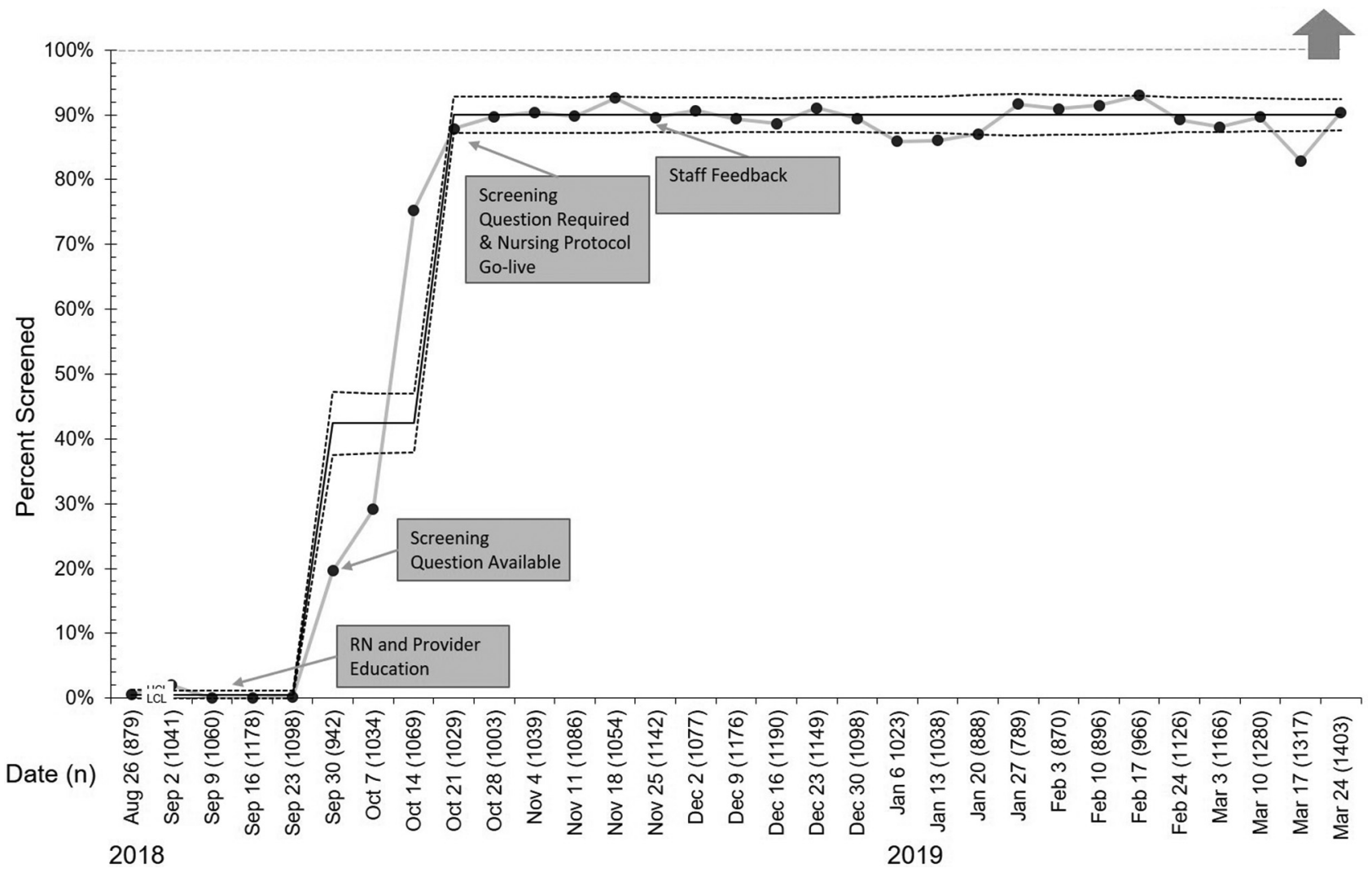

Abstract 4 Figure 1 


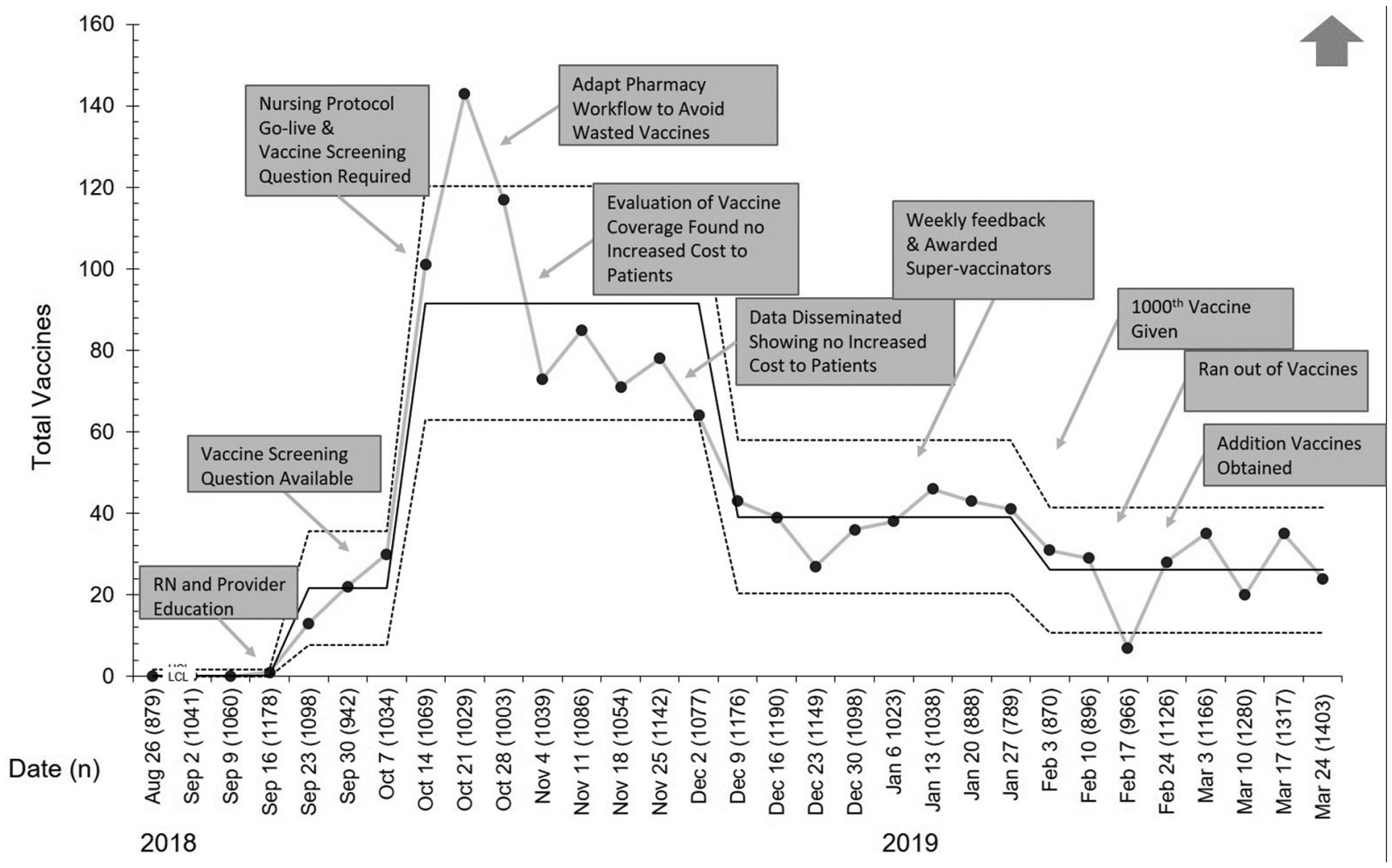

Abstract 4 Figure 2

\section{CREATIVITY OVER CAPITAL: USING QI TO ENSURE ACCESS}

Talia Buitrago, Sheetal Vora. Atrium Health Levine Childrens, US

\subsection{6/bmjoq-2019-ihi.5}

Background In 2016, our patients waited 3-5 months to be seen at our rheumatology clinic. This delay resulted in bad outcomes for some patients. Using creative problem solving, without financial outlay, our team achieved and sustained significant gains.

Objectives To improve the rate of appointments scheduled within 30 business days for patients requiring ongoing rheumatic care from $35 \%$ to $85 \%$.

Methods We used the Model for Improvement with aim statement and data management plan. We defined current

\section{Appointments Scheduled within 30 Business Days for Referred Patients Requiring P Chart Ongoing Care}
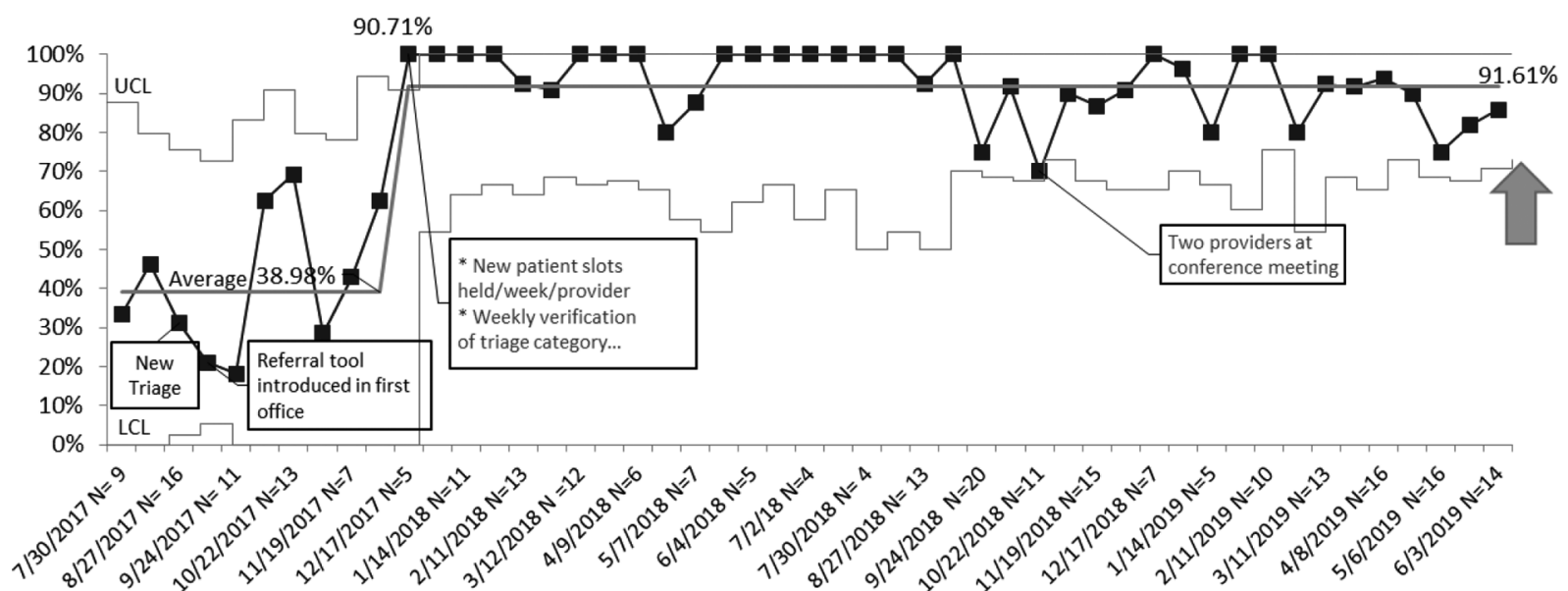

Abstract 5 Figure 1 